\title{
Hand-Raising Gesture Detection with Lienhart-Maydt Method in Videoconference and Distance Learning
}

\author{
Tiago S. Nazaré and Moacir Ponti \\ Instituto de Ciências Matemáticas e de Computação - Universidade de São Paulo \\ 13566-590 São Carlos, SP, Brazil \\ tiagosn@grad.icmc.usp.br moacir@icmc.usp.br \\ http://www.icmc.usp.br/ moacir
}

\begin{abstract}
In video-conference and distance learning videos, the moment that someone makes a hand-raising gesture is relevant to be included in the video annotation. However, gesture recognition can be challenging in such scenarios. We propose a system to detect faces, the hand-raising gesture and annotate the video. The Lienhart-Maydt object detection method is used, in which each frame is classified. Then, the gesture is detected by analyzing intervals of frames. Our approach was tested in videos with several characteristics. The results show that our method can deal with illumination and background variations, is able to detect multiple gestures and it is robust to confusing gestures. Besides it allow the use of moving cameras.
\end{abstract}

Keywords: Video processing, gesture detection, video annotation.

\section{Introduction}

Gesture recognition is a challenging task that is often addressed with complex sensors and methods, such as the use of depth sensors and multiple classifier systems, under controlled acquisition conditions [8]. Simpler methods, on the other hand, are not useful to specific applications since there is a lower concern about false positives and false negatives minimization. When the problem of gesture is more specific, it is possible to find more viable solutions. It is the case of hand-rising detection, applied to video-conference and distance learning videos to facilitate the annotation task.

In order to help the video annotation, we propose a system to detect faces and the hand raising gesture, i.e. an open hand raised, with the palm of the hand facing forward. A study is presented to investigate the robustness of already existing methods under several conditions and resolutions. A combination of methods is used, in special the improved Viola-Jones or Lienhart-Maydt method [6].

Kölsch e Turk [4, 5] showed that the object visual detector proposed by Viola and Jones [10, originally proposed to detect faces, could be used to detect hand poses. Later, Lienhart e Maydt [6] developed a more efficient method, based on 
the Viola-Jones original method, not used by Kölsch e Turk [4, [5] to evaluate their experiments.

We believe this investigation can shed a light about the use of this kind of method under conditions such as camera alternation, filming with a moving camera, and illumination changing such as when someone turn on/off the lights. The contributions of our study can be summarized in three parts: i) a method for a hand-raising gesture detection, ii) the study of the limitations of the Lienhart-Maydt method for this application, iii) and a new dataset of images to detect an open hand gesture.

\section{Related Work}

Among related work that specifically address this problem, Yao and Cooperstock [1] assumed that the heads of people in an audience are captured by the camera in a single horizontal line. It looks for movement and human skin in regions above the heads. When such events occur, a straight line is fitted using average points, if the slope of the line is between 45 and 135 degrees, a hand raising gesture is considered to be detected.

Duan and Liu [2] address the problem using indoor human silhouette analysis. It is able to work with moving people and groups. However the camera should remain still. The general pipeline of the method is foreground detection, followed by blob detection, candidate regions extraction (connected components located above the silhouettes), feature extraction using an R-transform and, finally a classification that looks for an arm or raising hand.

This study aims to improve the issues on those related work. We studied different acquisition conditions, including camera movement, changing in illumination, background and partial occlusion.

\section{Viola-Jones and the Improved Lienhart-Maydt Method}

The Viola-Jones method uses integral images and Haar-like operators to obtain several features. A boosting approach [3] is used to select a reduced number of visual descriptors to handle the problem. Finally, it uses a combination of classifiers in cascade, with increasing complexity. This cascade approach eliminates regions of low similarity, dedicating more effort on the classification of regions that are similar to the object of interest. Lienhart and Maydt [6] introduced two changes in the original algorithm: i) a new set of rotated Haar-like features [7] and ii) an improvement on the cascade classifiers based on a stage post-optimization scheme. The authors indicate an increase of $23.8 \%$ in the overall performance.

The Lienhart-Maydt [6] version of the object detector proposed by Viola and Jones [10] is used in this study to detect faces and hands in video frames, so that we can calculate the relative position between faces and hands and then detect a hand raising gesture, as described in next section. 


\section{Gesture Detection and Video Annotation}

\subsection{Method}

Using the Lienhart-Maydt method, we look for both faces and hands on each video frame. If it detects both faces and hands, we compute the relative position between them, to check if it is compatible with a hand raising gesture.

We consider a hand-raising gesture when a person raises his/her hand open next to his/her face. For this reason, the height of the hand should be at least in the line of shoulders. Thus, for each face the algorithm search for a hand surrounding the face. In order to reduce the search space, only a region proportional to two and a half (2.5) faces is considered to search for a hand in the horizontal direction, both right and left. In the vertical direction, the region proportional to three (3) faces above and half (0.5) face below. Those choices are explained by the average size of proportions face and arm in human beings, as depicted in Figure 1.

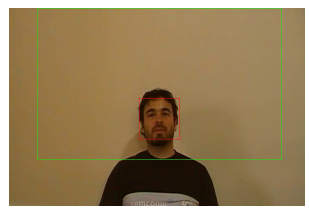

(a)

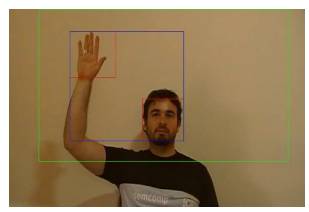

(b)

Fig. 1. Gesture search space: (a) face detected is showed using a red rectangle and the search space in a green rectangle; (b) hand detected is showed inside a red rectangle and the blue rectangle show the relation between hand and face.

Since the algorithm must detect both right and left hands, it should be trained with both right and left examples. In order to avoid this we trained only with left hands. To detect the right hands, we flip the image and than perform a second search.

A frame labeled as positive is those in which at least one pair face-hand satisfies the conditions cited before, otherwise it is considered negative. The Algorithm [1 summarizes the steps for the whole procedure.

After all frames are classified, we look for an interval of hand-face detections. A hand-raising gesture is detected inside a time interval when:

1. The duration of the gesture is at least 1 second;

2. The first and last frames are considered positive;

3. Between the first and last frames there are at least $80 \%$ of positive frames;

4. There are no sequences of negative frames with duration of more than 1 second of video. 


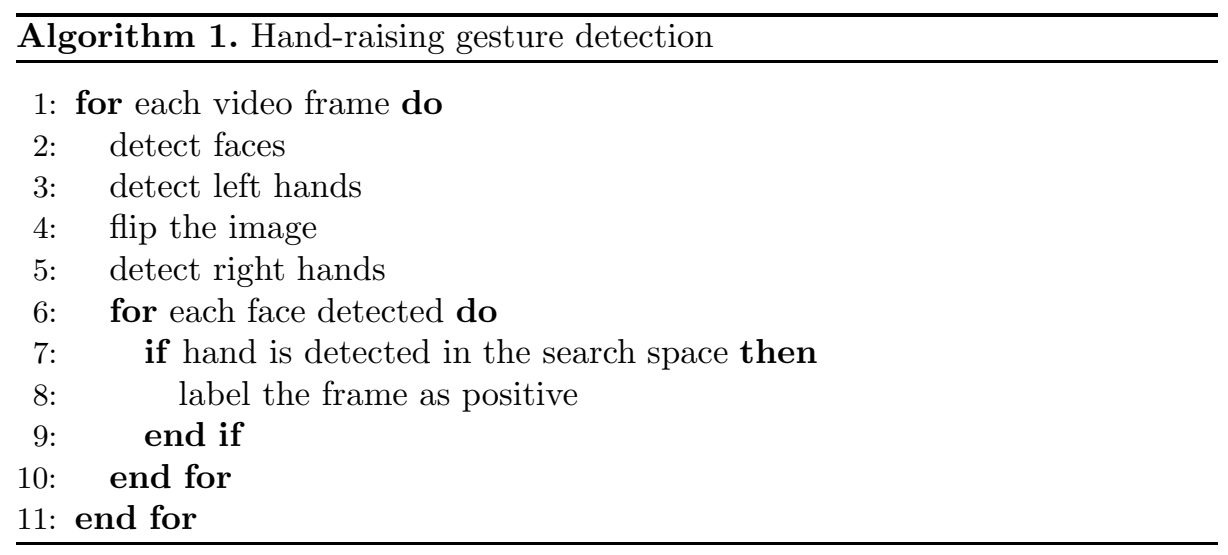

\subsection{New Training Hand Dataset and Implementation}

We used the OpenCV library version 2.1 [1] was used to implement the detector. This library has cascade classifiers trained to detect faces, available in XML files. It is also possible to create new cascade classifiers using positive and negative examples, and also store it in a XML file.

The face detection was performed using the already available classifier. To detect hands, we used 905 images of open left hands with different backgrounds, illumination variations, and changing finger positions (open and close). We also collected 1000 negative examples, both RGB and grayscale images (most of grayscale images were collected from [9]). In Figure 2 negative and positive examples are shown. The image dataset and the XML files are available in the project webpage 1 .

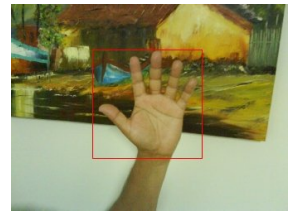

(a)

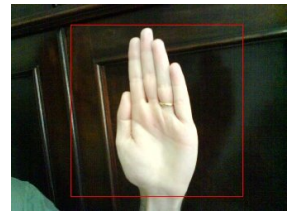

(b)

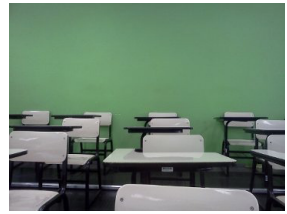

(c)

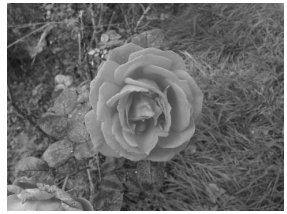

(d)

Fig. 2. Examples of images collected: (a-b) positive examples (c-d) negative examples

\section{$5 \quad$ Experiments}

A total of 16 videos were produced in order to test the method. The Table 1 summarizes the characteristics of each video. Three of those videos were recorded in a distance learning context (\# 5, 6 and 16), and the remaining in videoconferencing context. The resolutions indicated are: (A) 720x480, (B) 640x480,

\footnotetext{
${ }^{1}$ http://www.icmc.usp.br/ moacir/project/VideoProcessing/
} 
(C) $1280 x 720$. In order to check the robustness of the method, some situations were included in the videos, as indicated in Table 1:

1. Artificial illumination variation during the video (switching lights on and off, open a window, etc.);

2. Confusing gestures (scratch the head, spreading the arms, holding the head with an open hand, etc.);

3. External (natural) illumination;

4. Camera movement;

5. Multiple gestures (two or more people raising their hands simultaneously);

6. Partial occlusion of the hand and/or face;

7. Two or more people in different distances to the camera;

8. Variation of hand position (scale) in the same gesture;

Table 1. Characteristics of each video

\begin{tabular}{c|cccccccccccccccc}
\hline \hline Video ID & 1 & 2 & 3 & 4 & 5 & 6 & 7 & 8 & 9 & 10 & 11 & 12 & 13 & 14 & 15 & 16 \\
\hline \# Persons & 1 & 1 & 2 & 1 & 2 & 2 & 1 & 1 & 1 & 1 & 1 & 1 & 1 & 1 & 1 & 13 \\
Tests & 1 & 2 & 1,2 & 3,4 & 5,6 & 5 & 6,2 & 6,2 & 6 & 6,2 & 6,2 & 6 & $1,2,6$ & 1,8 & 2 & $4,5,7$ \\
Resolution & $\mathrm{A}$ & $\mathrm{A}$ & $\mathrm{A}$ & $\mathrm{A}$ & $\mathrm{A}$ & $\mathrm{A}$ & $\mathrm{B}$ & $\mathrm{B}$ & $\mathrm{B}$ & $\mathrm{B}$ & $\mathrm{B}$ & $\mathrm{B}$ & $\mathrm{B}$ & $\mathrm{B}$ & $\mathrm{B}$ & $\mathrm{C}$ \\
FPS & 29 & 29 & 29 & 29 & 29 & 29 & 24 & 25 & 24 & 24 & 24 & 24 & 25 & 15 & 30 & 29 \\
\hline \hline
\end{tabular}

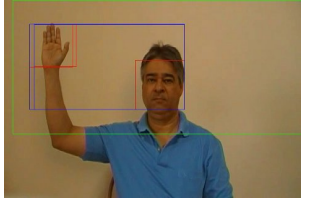

(a)

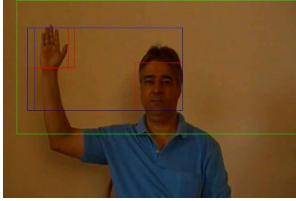

(b)

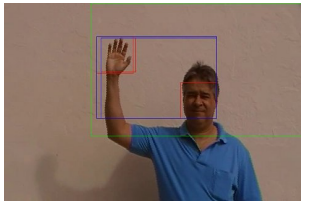

(c)

Fig. 3. Neighbor frames with sudden change in ambient lighting and camera movement (a) starting illumination condition (b) lights turned off (c) natural illumination and camera movement

Sudden changes in the illumination during the video are tested in videos \#1 and \#3. It significantly modifies the pixel values, which often is an issue in movement-based gesture detection [2]. An example of such change (lights turned off and natural illumination), as well as camera movement is shown in Figure 3 .

Another issue is the false detection of gestures that are close to a raising hand. During a class or videoconference a person might spread the arms, scratch the face or head, or do something that can be confused with a raising hand gesture. It happens in some videos such as in the examples of Figure 4. Besides, the most difficult problem to overcome when using the Lienhart-Maydt method is the occlusion and partial occlusion. Some examples are shown also in Figure 4.

In both videoconferencing and classroom contexts, there are often multiple gestures and people positioned in different distances in relation to the camera. The videos \#3, \#5, \#6 e \#16 include such scenarios as depicted in Figure 5 . 


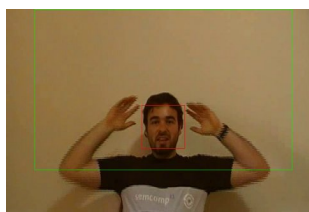

(a)

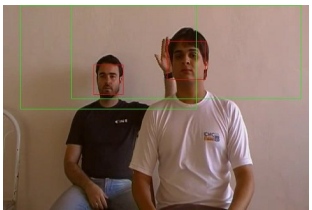

(b)

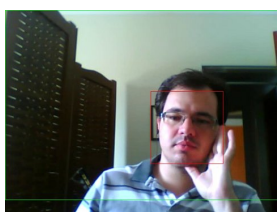

(c)

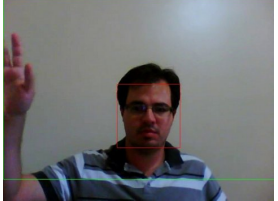

(d)

Fig. 4. Examples of confusing gestures (a) and (c), and partial occlusion (b) and (d)

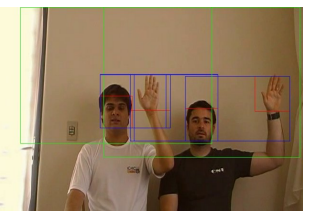

(a)

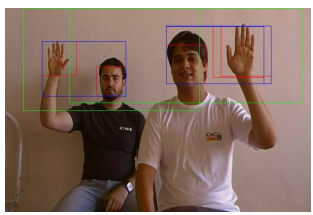

(b)

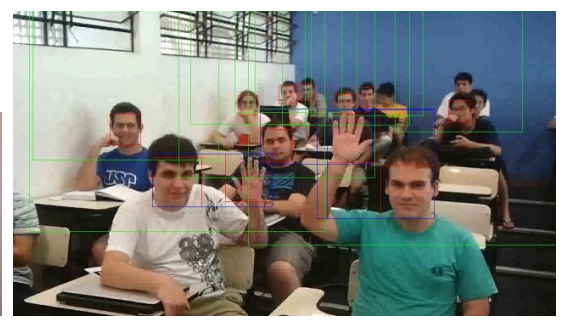

(c)

Fig. 5. Detected frames with multiple simultaneous gestures

\section{Results and Discussion}

The Table 2 shows, for each video, TP: true positive rate, FP: false positive rate, $F N$ : false negative rate, $T N$ : true negative rate, FPS: frames per second, precision, accuracy and running time (seconds).

All processing is performed offline, after the video is recorded, and the video annotated for future search.

The proposed method achieved the following robustness results:

- Illumination changes: did not affected the performance, including when the change occur during a gesture.

- Confusing gestures: did not affected the performance, the method seems to be very good on discharging false or confusing hand gestures.

- Occlusion: the method cannot handle partial occlusion in the external borders of the frame, since the detector cannot center a rectangle in the region. This is the reason why the videos \#7-13 have lower accuracy and precision values. For partial occlusion inside the frame, of both hands and faces, we observed that the method could not handle more than $15 \%$ of occlusion.

- Multiple gestures and scale variation: the method handle well multiple gestures, as indicates the results for videos \#5, \#6 and \#16. The scale is also not an issue if well managed. A case of failure is the video \#16 in which gestures are not detected when people are too far from the camera, as shown in Figure 5. For this reason, it is important to control the audience position in order to assure a good result.

- Camera movement: it did not affected the results, all gestures were detected under moderate movement. 
Table 2. Results

\begin{tabular}{cccccccccc}
\hline ID\# & TP & FP & FN & TN & FPS & Precision & Accuracy Running Time (s.) \\
\hline 1 & 838 & 4 & 10 & 811 & 29 & 0.9952 & 0.9915 & 545 \\
2 & 401 & 7 & 6 & 887 & 29 & 0.9828 & 0.9900 & 291 \\
3 & 1103 & 28 & 1 & 815 & 29 & 0.9752 & 0.9851 & 739 \\
4 & 699 & 11 & 7 & 403 & 29 & 0.9845 & 0.9839 & 707 \\
5 & 973 & 54 & 84 & 418 & 29 & 0.9474 & 0.9097 & 831 \\
6 & 429 & 29 & 18 & 363 & 29 & 0.9366 & 0.9439 & 348 \\
7 & 2 & 5 & 91 & 454 & 24 & 0.2857 & 0.8260 & 299 \\
8 & 68 & 8 & 355 & 712 & 25 & 0.8947 & 0.6824 & 627 \\
9 & 42 & 2 & 180 & 339 & 25 & 0.9545 & 0.6767 & 268 \\
10 & 112 & 0 & 182 & 254 & 24 & 1.0000 & 0.6678 & 151 \\
11 & 167 & 25 & 162 & 369 & 24 & 0.8697 & 0.7413 & 226 \\
12 & 35 & 1 & 223 & 167 & 24 & 0.9722 & 0.4741 & 179 \\
13 & 159 & 115 & 76 & 460 & 24 & 0.5802 & 0.7641 & 243 \\
14 & 234 & 0 & 55 & 69 & 15 & 1.0000 & 0.8463 & 128 \\
15 & 645 & 52 & 286 & 618 & 30 & 0.9253 & 0.7888 & 892 \\
16 & 168 & 27 & 94 & 114 & 29 & 0.8615 & 0.6997 & 805 \\
\hline
\end{tabular}

\section{Conclusion}

The Lienhart-Maydt method, used as basis for our method, was able to overcome many issues of previous works that tried to detect the same gesture, since it is robust to scale and illumination changes. The frame-by-frame analysis and the smoothness of the gesture detection in intervals is probably the cause of the success in other conditions such as camera movement and multiple gestures. Our method is not dependent on a specific camera or pose, can handle variations in illumination even during the gesture, is able to detect gestures with moving cameras, and work with different backgrounds and groups of people.

The drawbacks of the method include failure of detecting faces and hand with partial occlusion, and the necessity of filming the audience facing front, i.e., towards the camera, with small tolerance for angles (up to 15, as tested by video \#16). Also, the detector runs three times, to detect faces, right and left hands, hindering the possibility of online processing. For annotation purposes it is not an issue since it is often performed after the recording.

It is important to note that the method has flexibility to detect different objects, so it can be a good choice to help on semi-automatic annotation systems. To improve the running time, we suggest the use of GPUs to implement the detector. Also, a better occlusion treatment is a matter of future studies.

Acknowledgment. The authors are grateful to CNPq for the student scolarship and FAPESP (proc. n. 2011/16411-4) for supporting the project. 


\section{References}

1. Bradski, G.: The OpenCV Library. Dr. Dobb's Journal of Software Tools (2000)

2. Duan, X., Liu, H.: Detection of hand-raising gestures based on body silhouette analysis. In: IEEE Int. Conf. Robotics and Biomimetics, pp. 1756-1761 (2009)

3. Freund, Y., Schapire, R.E.: Experiments with a new boosting algorithm. In: Proc. 13th International Conference on Machine Learning (ICML 1996), pp. 148-156 (1996)

4. Kölsch, M., Turk, M.: Analysis of rotational robustness of hand detection with a viola-jones detector. In: Proceedings of the 17th International Conference on Pattern Recognition (ICPR 2004), vol. 3, pp. 107-110 (2004)

5. Kölsch, M., Turk, M.: Robust hand detection. In: 6th IEEE Int. Conf. on Automatic Face and Gesture Recognition, Seoul, Korea, pp. 614-619 (2004)

6. Lienhart, R., Maydt, J.: An extended set of haar-like features for rapid object detection. In: IEEE Int. Conf. Image Processing (ICIP), pp. 900-903 (2002)

7. Mohan, A., Papageorgiou, C., Poggio, T.: Example-based object detection in images by components. IEEE Transactions on Pattern Analysis and Machine Intelligence 23, 349-361 (2001)

8. Qin, S., Zhu, X., Yang, Y., Jiang, Y.: Real-time hand gesture recognition from depth images using convex shape decomposition method. Journal of Signal Processing Systems, 1-12 (2013)

9. Seo, N.: Tutorial: OpenCV Haar training (rapid object detection with a cascade of boosted classifiers based on Haar-like features) (2011), http://tutorial-haartraining.googlecode.com/svn/trunk/data/negatives/

10. Viola, P., Jones, M.: Rapid object detection using a boosted cascade of simple features. In: Proc. 2001 IEEE Conference on Computer Vision and Pattern Recognition (CVPR 2001), pp. 511-518 (2001)

11. Yao, J., Cooperstock, J.R.: Arm gesture detection in a classroom environment. In: Proc. 6th IEEE Work. Appl. Computer Vision, pp.153-157 (2002) 